\title{
ASSISTÊNCIA DOMICILIAR NA CIDADE DE SALVADOR-BA: POSSIBILIDADE DE ATUAÇÃO FONOAUDIOLÓGICA EM MOTRICIDADE OROFACIAL
}

\section{Home care in Salvador-BA: a work possibility for speech therapists in orofacial myofunction}

\author{
Sarah Leite Barros da Silva ${ }^{(1)}$, Rosana Inácio Protásio Musse ${ }^{(2)}$, Kátia Nemr ${ }^{(3)}$
}

\begin{abstract}
RESUMO
Objetivo: caracterizar o atendimento fonoaudiológico nas empresas de Assistência Domiciliar de Salvador. Métodos: pesquisa retrospectiva por meio da aplicação de questionário às empresas, fonoaudiólogos e pacientes atendidos com fonoaudiólogo, de Janeiro a Março de 2006. Resultados: o tempo médio de funcionamento das empresas é 9,17 anos. A média de pacientes atendidos por fonoaudiólogo é 14,33 com média de 3,17 fonoaudiólogos atendendo por empresa; profissionais presentes em $100 \%$ das empresas são médicos e fisioterapeutas; os fonoaudiólogos trabalham por prestação de serviço, com valor médio de 33 reais por atendimento. A maioria dos pacientes são mulheres, idosos (média de 69 anos), com diagnóstico de doença neurológica: AVE (39,1\%), Demências (19,1\%); os fonoaudiólogos são em maioria mulheres, jovens e graduadas no próprio estado. Conclusões: a assistência domiciliar é atividade recente na Bahia, porém a presença do Fonoaudiólogo já é estabelecida, com profissionais graduados no próprio estado e trabalhando por prestação de serviço.
\end{abstract}

DESCRITORES: Serviços de Assistência Domiciliar; Assistência Domiciliar; Pacientes Domiciliares; Transtornos de Deglutição; Fonoterapia

\section{INTRODUÇÃO}

Os pacientes, que até pouco tempo atrás eram tratados basicamente nos hospitais, são agora transferidos o mais rapidamente possível para seus domicílios, onde continuarão seus tratamentos, com programas de reabilitação, de recuperação ou de cuidados paliativos ${ }^{1,2}$.

$\mathrm{O}$ atendimento domiciliar consiste numa gama de serviços realizados no domicílio e destinados ao suporte terapêutico do paciente. Os principais objetivos deste tipo de atendimento são: contribuir para a otimização dos leitos hospitalares e do atendimento ambulatorial, visando à redução de custos;

(1) Fonoaudióloga da Reabilitar Health Care; Especializanda em Motricidade Orofacial.

(2) Fonoaudióloga Clínica da Due Comunicação e Desenvolvimento; Especializanda em Motricidade Orofacial

(3) Fonoaudióloga; Docente da Universidade de São Paulo, USP, São Paulo, SP; Coordenadoria de Serviços de Saúde da Secretaria de Estado de Saúde de São Paulo; Doutora em Psicologia Social pela Universidade de São Paulo. reintegrar o paciente em seu núcleo familiar e de apoio; proporcionar assistência humanizada e integral, por meio de uma maior aproximação da equipe de saúde com a família; estimular uma maior participação do paciente e de sua família no tratamento proposto; promover educação em saúde; ser um campo de ensino e pesquisa. Seus potenciais benefícios seriam a diminuição das re-internações e dos custos hospitalares, a redução do risco de infecção hospitalar e a manutenção do paciente no núcleo familiar e o aumento da qualidade de vida deste e de seus familiares ${ }^{1,2}$.

Estudos apontam o atendimento aos idosos e o cuidado domiciliar de longo prazo como sendo a "voz do futuro" na enfermagem ${ }^{3}$, uma vez que tem ocorrido aumento da expectativa de vida e incidência de doenças crônico-degenerativas ${ }^{4}$.

O conceito de Home Care ou Assistência Domiciliar teve início em 1880 nos Estados Unidos, com desenvolvimento mais expressivo a partir da década de 50. No Brasil, o Home Health Care foi introduzido apenas na década de 80 , havendo cerca 
de 30 empresas atualmente no país funcionando com equipes multidisciplinares, podendo incluir os seguintes profissionais: médico, enfermeiro, fisioterapeuta, fonoaudiólogo, terapeuta ocupacional, psicólogo, dentista, nutricionista, assistente social e técnicos/ auxiliares de enfermagem ${ }^{5}$.

Têm sido verificadas formas distintas de assistência: visita domiciliar, atendimento domiciliar e internação domiciliar. Sendo a visita domiciliar um atendimento realizado com objetivo de avaliar as necessidades do paciente, de seus familiares e do ambiente onde vivem, para estabelecer um plano assistencial voltado à recuperação e/ou reabilitação. São realizadas orientações às pessoas responsáveis pela continuidade do cuidado no domicílio; o atendimento domiciliar, no qual ocorrem atividades assistenciais exercidas por profissionais e/ou equipe de saúde na residência do cliente, para executar procedimentos mais complexos, que exigem formação técnica para tal; e a internação domiciliar que são atividades assistenciais especializadas, exercidas por profissionais e/ou equipe de saúde na residência do cliente, com oferta de recursos humanos, equipamentos, materiais e medicamentos, assemeIhando-se ao cuidado oferecido em ambiente hospitalar (instalação de um mini-hospital). A permanência de profissionais de enfermagem junto ao cliente é pré-estabelecida 6,12 ou 24 horas ${ }^{6-8}$.

Com relação à aplicação deste modelo de atendimento no setor público brasileiro, alguns autores entendem que um Programa de Internação Domiciliar (PID) representa a construção de nova lógica de atenção, com enfoque na promoção e prevenção à saúde, superando um modelo hegemônico centrado na doença, para construir um pensar e um fazer sustentado na produção social do processo saúde-doença ${ }^{9}$.

Foi sancionada em abril de 2002, pelo Ministério da Saúde, a Lei no 10.424 que estabelece, no âmbito do SUS, o atendimento e o PID (Programa de Internação Domiciliar). Essa Lei inclui, principalmente, os procedimentos médicos, de enfermagem, fisioterapêuticos, psicológicos e de assistência social, necessários ao cuidado integral dos usuários em seu domicílio ${ }^{10}$.

São considerados "candidatos" a este tipo de atendimento aqueles indivíduos impossibilitados de deixar sua casa sem esforço excepcional e apoio; os pacientes (nesta condição) recebem serviços de saúde em casa, incluindo tratamento médico e cuidado pessoal ${ }^{11}$.

Há algumas doenças que estão vinculadas a este tipo de atendimento: câncer, peneumonia, AIDS, insuficiência renal, ostomizados, pré-eclâmpsia, fratura de fêmur, DPOC (doença pulmonar obstrutiva crônica), ICC (insuficiência cardíaca congestiva),
AVE (acidente vascular encefálico), enfisema pulmonar, úlceras vasculares, diabéticas, entre outras ${ }^{5}$. Em se tratando da atuação fonoaudiológica em Home Care, os principais sintomas a serem tratados serão a disfagia e as afasias ${ }^{12}$.

Alguns autores restringem o "público comum" para este tipo de atendimento definindo-o como uma alternativa de modalidade de assistência à saúde para o tratamento de idosos com algum grau de dependência ${ }^{13}$.

Um estudo realizado com 42 pacientes de um programa de assistência domiciliária constatou que as mulheres representaram $80 \%$ da amostra, a idade média dos idosos foi de $82,73 \pm 6,45$ anos, $65 \%$ com idade superior a 80 anos. Os idosos apresentaram em média 6,10 $\pm 2,02$ comorbidades associadas, com maior presença de afecções músculoesqueléticas (90\%), neuropsiquiátricas $(87,5 \%)$ e cardiovasculares $(82,5 \%){ }^{14}$.

Em estudo realizado junto ao serviço de assistência domiciliar do Hospital São Francisco - Ribeirão Preto - SP, durante os doze meses de atividade do serviço de home care, houve predomínio de atendimento a clientes com faixa etária entre 70-80 anos, sexo feminino, com doenças neurológicas. Fizeram parte da equipe: profissionais de enfermagem, de psicologia, de nutrição, de fisioterapia e de medicina ${ }^{6}$.

$\mathrm{Na}$ Bahia, a Fonoaudiologia surgiu há 20 anos com profissionais oriundos de outros estados do Brasil, sendo que o primeiro curso de graduação foi iniciado em $1999{ }^{12}$.

É possível constatar que oferecer serviços de saúde em domicílio é uma tendência da atualidade em que a fonoaudiologia tem participação ainda discreta, com escasso material publicado. A realização desta pesquisa também se justifica por permitir análise e possibilidades de inserção fonoaudiológica mais expressiva na área de atuação domiciliar.

O objetivo desta pesquisa é caracterizar o atendimento fonoaudiológico nas empresas de Assistência Domiciliar na cidade de Salvador.

\section{MÉTODOS}

Trata-se de uma pesquisa com dois perfis: retrospectivo a partir de dados coletados em todas as empresas de Assistência Domiciliar que tinham fonoaudiólogo em seu quadro, na cidade de Salvador; e transversal observacional por meio da aplicação de questionário (Figura 1). Esses dados referiram-se à própria empresa, aos fonoaudiólogos a elas vinculados e aos pacientes a que assistem. A primeira parte do questionário foi aplicada nas empresas para coleta de dados destas e dos pacientes. A segunda parte foi aplicada com os fonoaudiólogos 


\section{Questionário}

\section{PARTE I}

- Dados da empresa:

1. Tempo de funcionamento:

2. Quantos pacientes estão em atendimento no geral?

3. Quantos pacientes estão em atendimento com fonoaudiólogo?

4. Quantos fonoaudiólogos atendem pela empresa?

5. Que outros profissionais fazem parte da empresa

6. Tipo de vínculo trabalhista:

7. Valor do atendimento fonoaudiológico:

- Dados do paciente:

1. Nome:

2. Gênero:

3. Idade:

4. Diagnóstico Médico:

5. Convênio:

\section{PARTE II}

- Dados do fonoaudiólogo:

1. Nome:

2. Idade:

3. Tempo de graduação e Universidade que cursou:

4. Maior titulação:

5. Há quanto tempo realiza atendimento domiciliar:

6. Alterações fonoaudiológicas de cada paciente descrito pela empresa

Figura 1 - Questionário para captação de dados das empresa, fonoaudiólogos e pacientes

para obtenção de dados pessoais e referentes às alterações fonoaudiológicas de cada paciente descrito pela empresa. Estas alterações foram classificadas de acordo com as seguintes áreas: cognição, voz, fala, linguagem e deglutição, sendo solicitado aos fonoaudiólogos que apontassem tantas alterações quantas o paciente apresentasse.

Os dados são referentes ao período de Janeiro, Fevereiro e Março do ano de 2006.

A presente pesquisa foi avaliada e aprovada pelo Comitê de Ética em Pesquisa do CEFAC - Saúde e Educação, sob no 26/06 e o considerou aprovado sem risco com necessidade do Termo de Consentimento Livre e Esclarecido.

Os resultados estão apresentados na forma de estatística descritiva com frequências absolutas e relativas e, quando possível, medidas de tendência central.

\section{RESULTADOS}

$\mathrm{Na}$ cidade de Salvador há seis empresas de assistência domiciliar. Verificou-se que dessas, 2
$(33,3 \%)$ são do tipo Atendimento Domiciliar (AD), $2(33,3 \%)$ são do tipo Internação Domiciliar (ID) e $2(33,3 \%)$ atuam em ambas as formas (AD e ID) (Figura 2). Não foram encontrados registros da adoção desta modalidade de atendimento pelo SUS (Sistema Único de Saúde) no município de Salvador.

Quanto ao tempo de funcionamento, observa-se que o tempo médio é de 9,17 anos. A empresa que atua há menos tempo tem quatro anos de funcionamento e a mais antiga 20 anos.

Com relação ao número de pacientes atendidos no total, as empresas de $A D$ tiveram uma média de admissões de 168,50 pacientes, enquanto as de ID, 65,75 pacientes, no mesmo período (Tabelas 1 e 2).

O número médio de pacientes submetidos a fonoterapia é 14,33 , com média de 3,17 fonoaudiólogos atendendo por empresa (Tabelas 3 e 4).

Quanto aos demais profissionais que compõem o quadro clínico, o Médico e o Fisioterapeuta estão presentes em todas elas (6) $100 \%$. O Enfermeiro em segundo lugar atende em (5) 83,3\%; Assistentes 


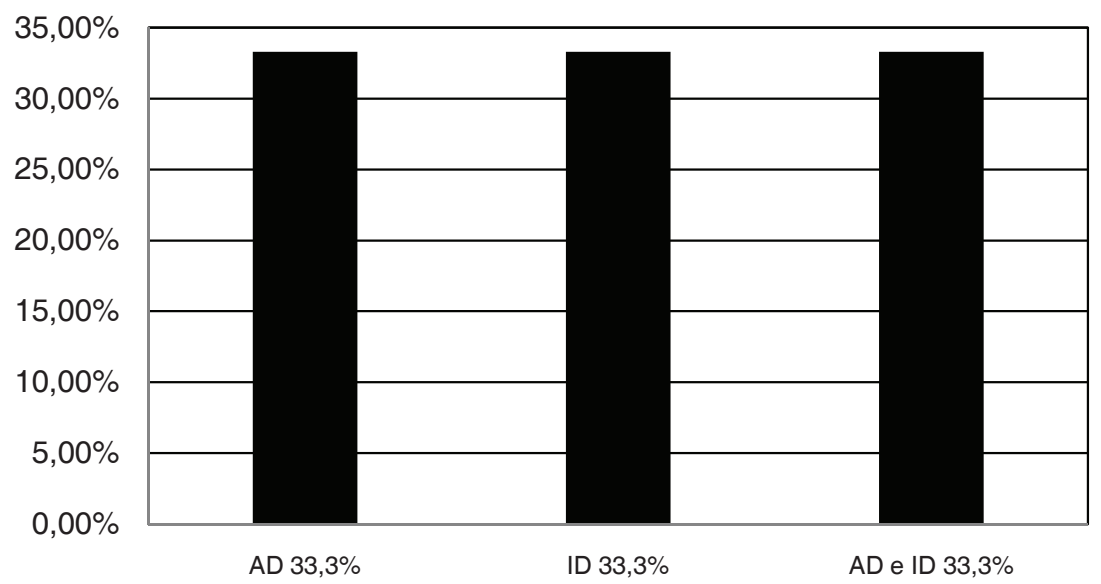

Figura 2 - Distribuição em percentuais dos tipos de atendimentos

Tabela 1 - Medidas de tendência central relativas a admissões das empresas de atendimento domiciliar

\begin{tabular}{c|c}
\hline Média & $\mathbf{1 6 8 , 5}$ \\
Mediana & 133,5 \\
Desvio padrão & 133,6 \\
Mínimo & 57 \\
Máximo & 350 \\
\hline
\end{tabular}

Tabela 2 - Medidas de tendência central relativas às admissões das empresas de internação domiciliar

\begin{tabular}{c|c}
\hline Média & 67,7 \\
Mediana & 64,5 \\
Desvio padrão & 38,26 \\
Mínimo & 26 \\
Máximo & 108 \\
\hline
\end{tabular}

Tabela 3 - Medidas de tendência central relativas ao número de pacientes em atendimento fonoaudiológico por empresa

\begin{tabular}{c|c}
\hline Média & $\mathbf{1 4 , 3}$ \\
Mediana & 13 \\
Desvio padrão & 8,5 \\
Mínimo & 6 \\
Máximo & 30 \\
\hline
\end{tabular}

Sociais e Nutricionistas trabalham em (4) $66,7 \%$ e o profissional Psicólogo em (3) $50 \%$. Os auxiliares de enfermagem estão presentes em (2) $33,3 \%$ e o Terapeuta Ocupacional em (1) $16,7 \%$ (Figura 3).
Tabela 4 - Medida de tendência central relativa à quantidade de fonoaudiólogos por empresa

\begin{tabular}{c|c}
\hline Média & $\mathbf{3 , 1}$ \\
Mediana & 3,5 \\
Desvio padrão & 1,4 \\
Mínimo & 1 \\
Máximo & 5 \\
\hline
\end{tabular}

Tabela 5-Medidas de tendência central relativas à faixa etária dos pacientes

\begin{tabular}{c|c}
\hline Média & $\mathbf{6 9 , 0 2}$ \\
Mediana & 74 \\
Desvio padrão & 21,0 \\
Mínimo & 0,16 \\
Máximo & 99 \\
\hline
\end{tabular}

Quanto à remuneração, observou-se que não há Fonoaudiólogos contratados, todos são prestadores de serviço.

Foram analisados dados de 115 pacientes e verificou-se que a média da faixa etária é de 69 anos (Tabela 5). Quanto ao sexo, a maioria dos pacientes atendidos por fonoaudiólogos é do sexo feminino, representando $56,5 \%$ e do masculino, $43,5 \%$.

Quanto ao diagnóstico médico, identificou-se que a maioria tem como doença de base o Acidente Vascular Encefálico - AVE (45) 39,1\%, logo em seguida, a patologia mais frequente foram as Demências (22) 19,1\% e o Parkinson (11) 9,6\%. A doença que afetou (5) 4,3\% dos pacientes foi a associação AVE + ITR (Infecção de Trato Respiratório) e a (4) 3,5\% foi a PC (Paralisia Cerebral); 


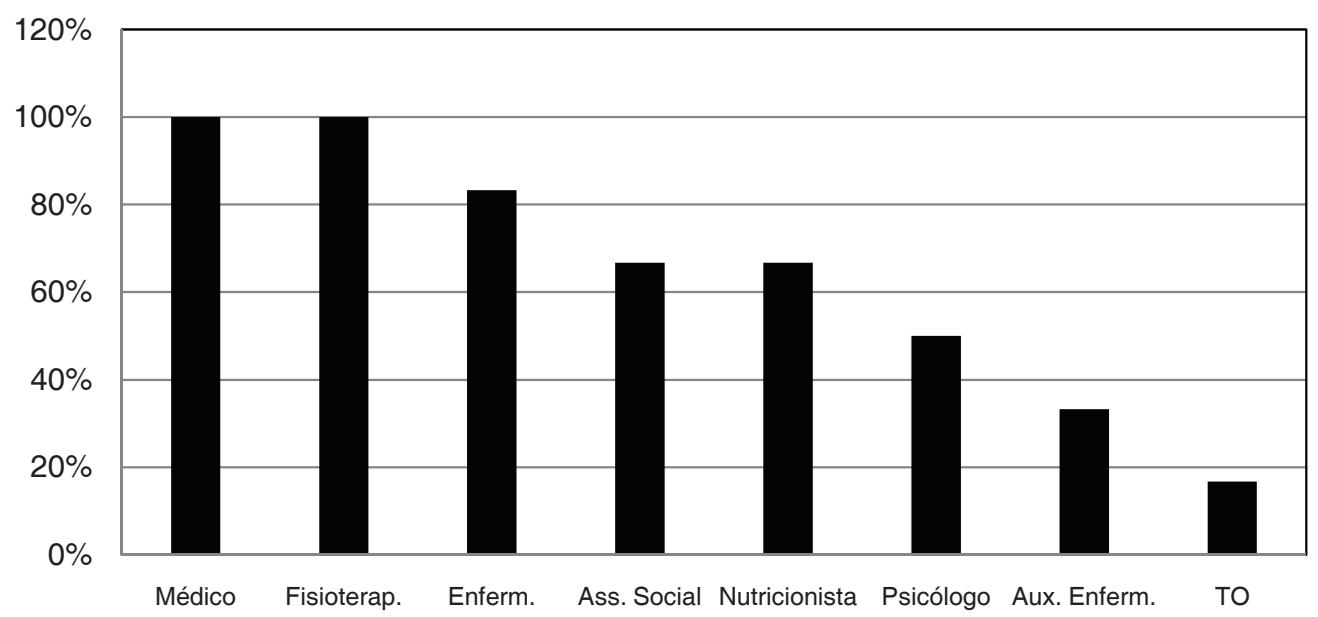

Figura 3 - Distribuição em percentuais do corpo clínico das empresas de assistência domiciliar

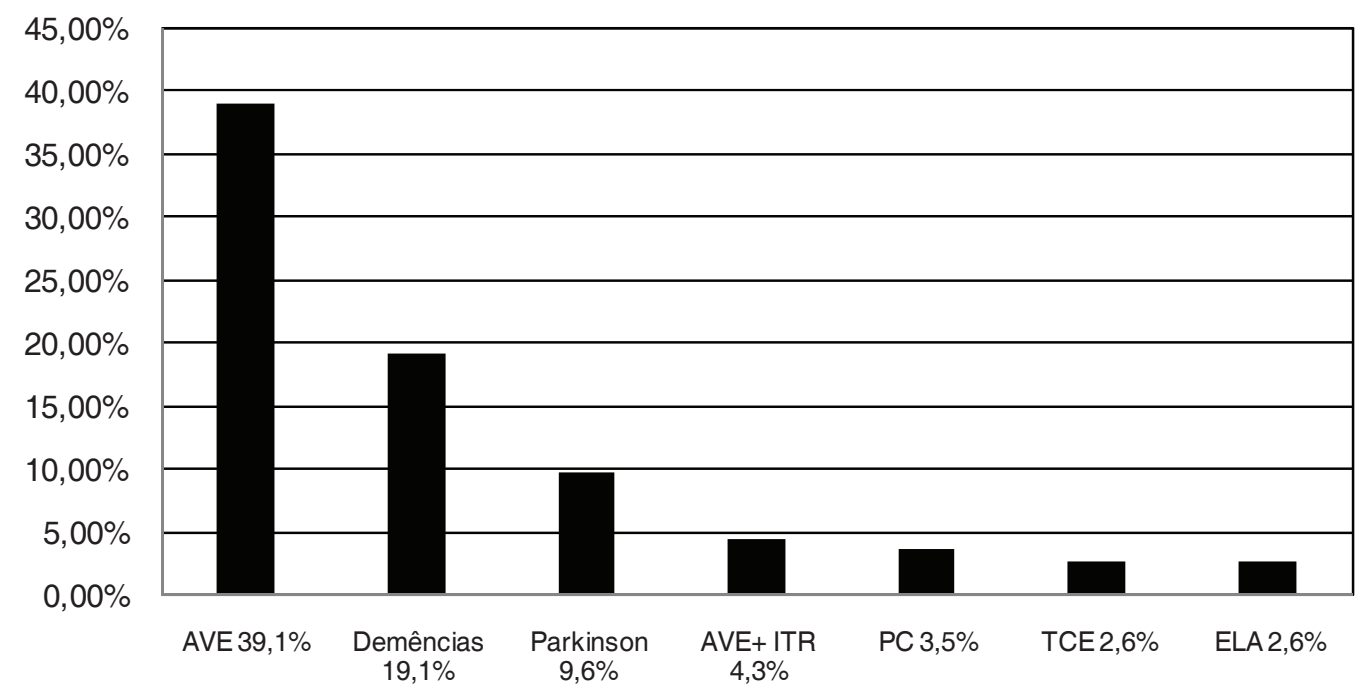

Figura 4 - Distribuição em percentuais do diagnóstico médico dos pacientes

as patologias, TCE (Traumatismo Crânio-encefálico) e ELA (Esclerose Lateral Amiotrófica) acometeram outros (3) 2,6\% cada (Figura 4); e (2) 1,7\% sofreram Parada Cardio-respiratória (PCR), AVE + Parkinson, Câncer de cabeça, Polineuropatia, ITR isoladamente. As patologias encefalopatia mitocondrial, câncer de pescoço, Síndrome de Arnold Choir, Doença pulmonar, Esclerose Múltipla, Distrofia Muscular, Estenose glótica e subglótica, fraqueza muscular, esquizofrenia, encefalite (herpes), Alzheimer + Parkinson e PCR + Câncer acometeram cada uma apenas (1) 0,9\% indivíduo da amostra.

Após análise dos dados constatou-se que a combinação de alterações fonoaudiológicas mais frequente era de Cognição-Linguagem-Deglutição (Figura 5).

Quanto aos convênios que oferecem cobertura à assistência domiciliar constatou-se que a Petrobrás é a maior fonte de encaminhamentos sendo responsável por (59) $65,6 \%$ dos atendimentos; logo após vem o convênio Santa Saúde com (10) 11,1\%, seguido do Cassi com (7) 7,8\%, o da Caixa Econômica Federal com (3) 3,3\% e o Sul América, com (2) $2,2 \%$. Os demais convênios não somam isoladamente $1 \%$ das indicações, sendo responsáveis por apenas 1 paciente; são eles: Ministério Público, Sesef, Unimed, Fusex, Norclínicas, Bradesco, Fassincra, Promédica e Casseb.

Com relação aos dados dos Fonoaudiólogos verificou-se que atuam apenas 15 profissionais vinculados a empresas em Salvador. Destes, 93,3\% (14) são do sexo feminino e $6,7 \%$ (1) do masculino. A idade média destes profissionais é de 27,20 anos.

Quanto ao tempo de graduação destes Fonoaudiólogos, observa-se que o tempo médio é de 4,47 anos. 


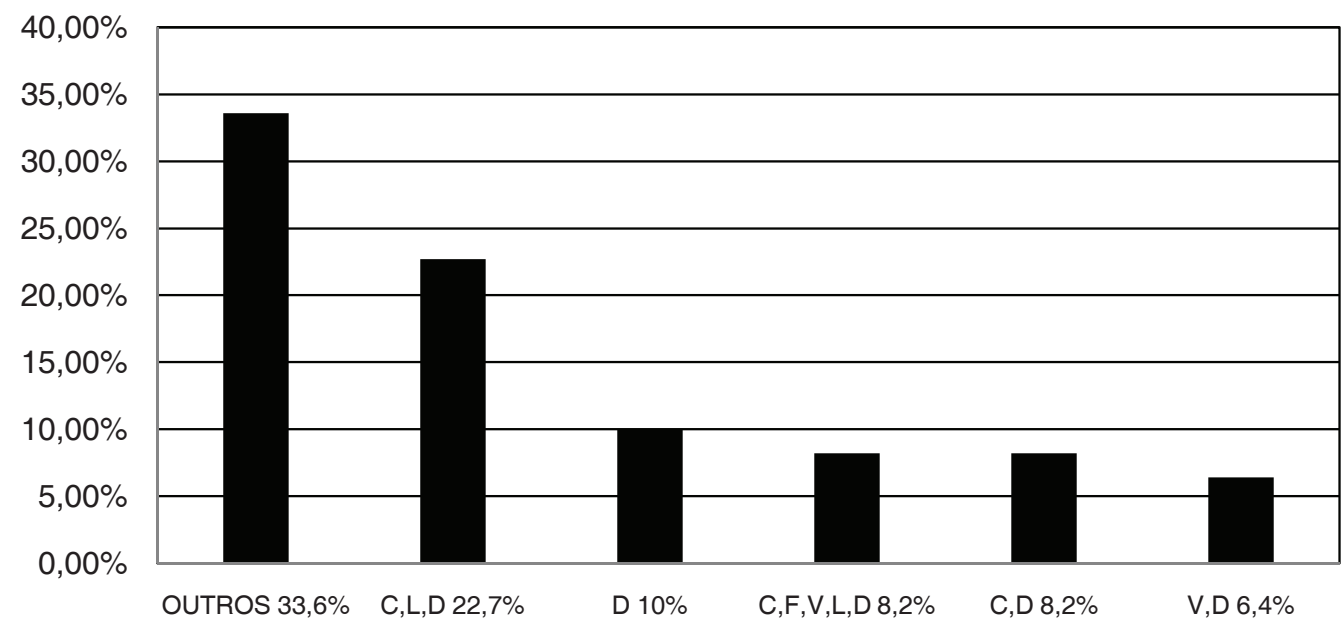

Figura 5 - Distribuição em percentuais de sintomas fonoaudiológicos apresentados pelos pacientes

Em relação ao local de formação, verificou-se que a maior parte dos Fonoaudiólogos, (6) 40\%, formaram-se na Universidade Federal da Bahia (UFBA), enquanto (4) $26,7 \%$ vieram da Universidade Católica de Pernambuco (UNICAPE). As demais Universidades foram o local de graduação de apenas 1 fonoaudiólogo (6,7\%). São elas: Pontifícia Universidade Católica de Campinas-SP (PUC-Campinas), Universidade de Marília - SP (UNIMAR), Universidade São Camilo-SP, Universidade do Estado da Bahia (UNEB), Universidade de Alfenas-MG (UNIFENAS).

Em relação à maior titulação (ainda que em curso), observou-se que (3) 20\% são apenas graduados; (1) $6,7 \%$ têm aprimoramento em Voz e MO; outros (3) $20 \%$ apresentam especialização em Fonoaudiologia Hospitalar, (2)13\% especialização em Gerontologia e (2) $13 \%$ em Motricidade Orofacial; (1) $6,7 \%$ especialização em Audiologia, outros (1) 6,7\% em Linguagem e outros (1) $6,7 \%$ em Saúde Pública. Apenas $6,7 \%$ (1) ingressaram em Mestrado. O tempo médio de atuação nesta área é de 2,7 anos. O menor tempo foi de 5 meses e o maior de 8 anos.

\section{DISCUSSÃO}

O achado de tempo médio de 9,17 anos é compatível com pesquisas que relatam o crescimento significativo desta atividade apenas na última década ${ }^{1}$. Apontava-se em 2001 a existência de cerca de 30 empresas deste ramo em todo o Brasil ${ }^{4}$, momento em que 5 das 6 empresas desta pesquisa já existiam demonstrando boa penetração desta atividade na cidade de Salvador.

Apesar de estar previsto na Lei $10.424^{7}$ a adoção desta modalidade de atendimento pelo SUS, desconhece-se que esteja sendo realizado no município de Salvador. Há registro desta implantação nas cidades de Marília, Londrina e Santos ${ }^{6}$, demonstrando a possibilidade de aplicação. O resultado é o de que não encontramos empresas domiciliares no SUS em Salvador, só particulares.

Em pesquisa realizada em Ribeirão Preto, num período de um ano foram captados 240 pacientes. Observou-se a presença dos profissionais médico, enfermeiro (incluindo auxiliares), nutricionista, fisioterapeuta e psicólogo ${ }^{5}$. Outra pesquisa apontou enfermeiros, fisioterapeutas, terapeutas ocupacionais, fonoaudiólogos e assistentes sociais ${ }^{11}$. A lei de n. 10.424 sancionada em Abril de 2002 prevê este atendimento pelo SUS contando com os seguintes profissionais: médico, enfermeiro, fisioterapeuta, psicólogo e assistentes sociais ${ }^{6}$. Outro artigo sobre $\mathrm{o}$ atendimento fonoaudiológico em home care cita médico, enfermeiro, fonoaudiólogo, fisioterapeuta, terapeuta ocupacional, psicólogo, dentista, nutricionista, assistente social e auxiliar de enfermagem ${ }^{4}$.

Pode ser observada a ausência de menção ao fonoaudiólogo em artigos brasileiros de outras áreas. Não há outros profissionais atuando em Salvador que não tenham sido mencionados neste artigo, bem como não há profissional citado com parte integrante da equipe que não esteja presente em ao menos uma das empresas de Salvador.

Quanto ao sexo dos pacientes, em estudo similar, confirmou-se a maior frequência do sexo feminino $58 \%$ contra $42 \%$ do masculino. Segundo o autor, acredita-se que esta diferença ocorra por uma expectativa de vida em 5 anos a mais das mulheres em relação aos homens ${ }^{5,11}$. Uma incidência ainda maior aparece em outro estudo onde os resultados apontam $80 \%$ de mulheres ${ }^{10}$, contra $20 \%$ dos homens. Defende-se que isto se deva ao fato de os homens estarem sujeitos a trabalhos de maior risco ${ }^{15}$.

Com relação à faixa etária, há concordância que a maioria dos pacientes domiciliares é composta de 
idosos. Um estudo encontrou média de idade de $82,7+/-5,4$ anos, sendo que $65 \%$ com idade superior a 80 anos ${ }^{10}$, outro aponta maioria com idade entre $70-80$ anos ${ }^{5}$ e outro confirma média etária semelhante $50-69$ anos ${ }^{11}$.

O estudo de Ribeirão Preto encontrou em sua amostra maior prevalência de doenças neurológicas, predominando Parkinson e Alzheimer, seguidos de neoplasias ${ }^{5}$. Desta forma, concorda com esta pesquisa no sentido de que de fato são mais prevalentes afecções neurológicas; no entanto o achado deste trabalho é de que o AVE ocorreu em significativa maioria dos pacientes atendidos. Outro estudo, com diferente classificação, identificou maior presença de afecções músculo-esqueléticas, seguida de neuro-psiquiátricas e cardiovasculares ${ }^{10}$.

Artigo sobre atuação de fonoaudiólogos em Home Care cita que as principais áreas de atuação deste profissional são a disfagia e a afasia ${ }^{4}$. 0 presente estudo apontou alteração cognitiva incluída na combinação de alterações mais frequentes e a presença das Disartrias e Disfonias.

O achado de que a maioria dos fonoaudiólogos é do sexo feminino mantém o histórico desta ciência mais estudada por mulheres. Esta maioria é jovem, sendo contrária a outro estudo em que a média etária dos profissionais da empresa de home care era de 44 anos, com 17 anos de experiência e sem especializações ${ }^{11}$.

Os dados revelaram um novo cenário na Bahia, em que a maioria dos profissionais em determinada área da fonoaudiologia é formada no próprio estado; isto porque a primeira turma graduou-se em 2003 (UFBA). Até então só atuavam em Salvador profissionais oriundos de outros estados do país ${ }^{12}$.
Profissionais vindos de Pernambuco constituem percentual significativo nesta área, conforme mostra o gráfico (Figura 5).

$O$ dado de que a significativa maioria finalizou ou está em curso de especialização revela um corpo de profissionais menos voltado à academia e em busca de um aprimoramento técnico. As três áreas das especializações mais referidas também revelam estreita ligação com a atuação: fonoaudiologia hospitalar, gerontologia e motricidade oral.

O tempo médio de atuação com assistência domiciliar é pequeno. Isto se deve à entrada de profissionais graduados em Salvador, que saíram de suas universidades a partir de 2003 e vêm ocupando espaço de maioria nas empresas de assistência domiciliar.

Esta pesquisa mostra relevância em traçar um panorama da assistência domiciliar com enfoque nas possibilidades de atuação fonoaudiológica, sendo instrumento norteador de quem deseje trabalhar nesta área ou comparar com outros serviços. Constitui-se também como dado oficial (não empírico) para correlações futuras ou presentes e de outros municípios, uma vez constatada a escassez de material publicado neste tema.

\section{CONCLUSÃO}

Os pacientes atendidos são em maioria mulheres, idosas, com diagnóstico de alteração neurológica e acometimento da Cognição-Linguagem-Deglutição e da Deglutição. As empresas de assistência domiciliar têm boa penetração na cidade de Salvador, com inserção significativa do profissional fonoaudiólogo como prestador de serviço.

\begin{abstract}
Purpose: to characterize the speech therapy service of home care service companies in Salvador. Methods: a partially retrospective survey through questionnaires done by companies, speech therapists and patients attended by speech therapist from January to March 2006. Results: companies have been open an average of 9.17 years. The average number of speech therapists per company is 3.17 and the average number of patients attended by speech therapist is $14.33 ; 100 \%$ of the company professionals are licensed doctors and physical therapists; speech therapists work as service rendering self-employed professionals, with average cost of $\mathrm{R} \$ 33,00$ per attendance. Most patients are women, elderly (average of 69 years), with diagnosis of neurological illness: Cerebrovascular disease (39.1\%), Dementia (19.1\%); most of the speech therapists are young women, who graduated from a local school. Conclusions: home care service is a recent activity in Bahia. However, speech therapist presence is already settled, with professionals graduated in their own states and working as service rendering self-employed professionals.
\end{abstract}

KEYWORDS: Home Care Services; Home Nursing; Homebound Persons; Deglutition Disorders; Speech Therapy 


\section{REFERÊNCIAS}

1. Floriani CA, Schramm FR. Atendimento domiciliar ao idoso: problema ou solução? Cad Saúde Pública. 2004; 20(4):986-94.

2. Perroca MG, Ek AC. O modelo sueco de home care avançado: organização e implicações da adoção desta modalidade de cuidado pelo serviço de saúde brasileiro. Rev Latino-Am Enferm. 2004; 12(6):851-8.

3. Tschudin V. The future nursing voice. Rev LatinoAm Enferm. 2003; 11(4):413-9.

4. Fialho AVM, Pagliuca LMF, Soares E. Adequação da teoria do déficit de autocuidado no cuidado domiciliar à luz do modelo de Barnum. Rev LatinoAm Enferm. 2002; 10(5):715-20.

5. Marlière GLL, Rios IJA, Sguizzardi A. Atendimento fonoaudiológico em "Home Care". In: Hernandez AM, Marchesan I. Atuação fonoaudiológica no ambiente hospitalar. São Paulo: Revinter; 2001. p. 169-75.

6. Fabrício SCC, Wehbe G, Nassur FB, Andrade JI. Assistência domiciliar: a experiência de um hospital privado do interior paulista. Rev Latino-Am Enferm. 2004; 12(5):721-6.

7. Núcleo Nacional das empresas de assistência domiciliar (NEAD). Disponível em: URL: http://www. neadsaude.org.br/assdomiciliar_conceitos.htm 8. Bolonhezi A, De Fina Júnior $E$, Ramão JE. Manual: entendendo a assistência domiciliar. São Paulo: Copyright; 2003.
9. Silva KL, Sena R, Leite JCA, Seixas CT, Gonçalves AM. Internação domiciliar no Sistema Único de Saúde. Rev Saúde Públ. 2005; 39:3.

10. Brasil. Lei 10.424, de 15 de abril de 2002. Acrescenta capítulo e artigo à Lei 8080, de 19 de setembro de 1990, que dispõe sobre as condições para a promoção proteção recuperação da saúde, a organização e funcionamento de serviços correspondentes e dá outras providências, regulamentando a assistência domiciliar no Sistema Único de Saúde. Brasília (DF): Diário Oficial da República Federativa do Brasil; 16 abr 2002. p. 1.

11. Descritor "pacientes domiciliares" Disponível em: URL: http://http://decs.bvs.br/cgi-bin/wxis1660. exe/decsserver/ Acesso em 24 jan 2006.

12. Cardoso C, Abreu TT. A fonoaudiologia na Bahia: uma história recente. Rev Baiana Saúde Públ. 2004; 28(1):96-9.

13. Duarte YAO, Diogo MJD. Atendimento domiciliar: um enfoque gerontológico. São Paulo: Atheneu; 2000. p. 3-17.

14. RicciNA, Kubota MT, Cordeiro RC. Concordância de observações sobre a capacidade funcional de idosos em assistência domiciliar. Rev Saúde Públ. 2005; 39(4):655-62.

15. Douglas CR.Envelhecimento: características e fisiologia geral. In: Douglas CR. Tratado de fisiologia aplicada à fonoaudiologia. São Paulo: Robe Editorial; 2002. p. 731-57.

DOI: 10.1590 / S1516-18462009005000001

RECEBIDO EM: 09/09/2007

ACEITO EM: 25/06/2008

Endereço para correspondência

Sarah Leite Barros da Silva

Rua Celeste, 10

Salvador - BA

CEP: 40430-010

E-mail: sarahfono@ hotmail.com 